\title{
Role of saline infusion sonography in abnormal uterine bleeding
}

\author{
Megha Sharma ${ }^{1}$, Shubham Joshi ${ }^{2}$, Akash Sharma ${ }^{3}$, Oby Nagar ${ }^{1}$, \\ Rakesh Khuteta $^{1}$, Meenu Bagarahatta ${ }^{4}$
}

\author{
${ }^{1}$ Department of Obstetrics and Gynaecology, ${ }^{2}$ Department of Medicine, ${ }^{4}$ Department of Radiodiagnosis, SMS Medical \\ College, Jaipur, Rajasthan, India \\ ${ }^{3}$ Department of Pediatrics, Dr. S.N. Medical College, Jodhpur, Rajasthan, India
}

Received: 16 September 2013

Accepted: 24 September 2013

\author{
*Correspondence: \\ Dr. Megha Sharma, \\ E-mail:meg_med5@yahoo.co.in
}

(C) 2013 Sharma M et al. This is an open-access article distributed under the terms of the Creative Commons Attribution Non-Commercial License, which permits unrestricted non-commercial use, distribution, and reproduction in any medium, provided the original work is properly cited.

\begin{abstract}
Background: To describe the diagnostic efficacy of Saline Infusion Sonography (SIS) in Abnormal Uterine Bleeding (AUB).

Methods: This is a hospital based observational descriptive study conducted in 121 women visiting the OPD with complain of AUB in any form, between September 2011-August 2012. SIS was done using Crooke's cannula followed by Diagnostic Hysteroscopy (DH) the following day. Results obtained were analysed.

Results: $61 \%$ of the cases of AUB were diagnosed to have some abnormality by hysteroscopy as compared to $51 \%$ by SIS. The sensitivity of SIS was greatest (100\%) for Mullerian anomalies, $75 \%$ for endometrial hyperplasia, $69 \%$ for endometrial polyps, $68.75 \%$ for submucous myomas and $66.67 \%$ for endometritis. The specificity was greatest for endometrial hyperplasia and adhesions (100\%), 99\% for submucous myomas, $98.3 \%$ for endometritis, $95.7 \%$ for endometrial polyps and mullerian anomalies. It had a $100 \%$ positive predictive value for endometrial hyperplasia, $92 \%$ and $83 \%$ for submucous myomas and endometrial polyps respectively. The overall sensitivity and specificity of SIS in our setting was $67.56 \%$ and $93.6 \%$ respectively. The diagnostic efficacy of SIS for Endometrial hyperplasia, Endometritis, Endometrial Polyps, Mullerian abnormalities, Submucus fibroid and Adhesions was 96.69\%, $97.53 \%, 89.25 \%, 95.86 \%, 95.04 \%$ and $98.34 \%$ respectively.

Conclusions: We found Saline Infusion Sonography to be an easy and safe procedure, though being less sensitive, it cannot replace hysteroscopy as a whole. Though hysteroscopy is a gold standard, saline infusion sonography is an easy, safe procedure for diagnosing intracavitary pathologies in low resource setting and despite being less sensitive, it can still reduce the number of hysteroscopies.
\end{abstract}

Keywords: Efficacy, SIS, AUB, Crooke's, Hysteroscopy

\section{INTRODUCTION}

The monthly changes in the hormonal, psychological, and physical milieu of a female in themselves are an example of dexterity and complexities. Since antiquity, the temporal relationship between the menses and the lunar phases has inspired names for menstruation, such as the "period". The regularity of menses was easily appreciated by the ancients, even if they had no understanding of its cause or purpose.
Any female with bleeding per vaginum outside her normal cyclical menses is said to have abnormal uterine bleeding.

AUB is the single most common complain that reproductive age women bring to the clinic. It is a symptom and not a disease. In women of child bearing age, AUB includes any change in menstrual period frequency or duration, or amount of flow, as well as bleeding between cycles. 
Hysteroscopy combined with guided biopsy has been considered as 'gold standard' in uterine cavity evaluation for AUB. The search for a procedure which is less invasive, cost effective and accurate in diagnosis led to the advent of endometrial imaging by using saline as a contrast medium (SIS). First described by Nannin et al in 1981, it was Richman ${ }^{1}$ in 1984 who used this technique first for evaluation of tubal patency in infertile women and the procedure was called as sonosalpingography.

Most of the studies conducted so far are from western developed countries and there is paucity of data from developing countries especially India. So this study was an attempt to find out an easier, better, more cost effective, less time consuming and an efficient diagnostic modality, with minimal morbidity in patients of AUB, as Hysteroscopy though is a gold standard, requires trained personnel, adequate equipment and infrastructure, and is associated with anaesthesia and other surgical complications, hospital stay and patient discomfort, which makes the procedure difficult to perform in all patients of AUB in a low resource setting.

\section{METHODS}

The study protocol was approved by the Regional Committee for Medical Ethics and Research. It was a hospital based observational descriptive study conducted in the Department of Obstetrics and Gynecology, SMS Medical College and Hospital, Jaipur between September 2011-August 2012. 126 females between 2445 years of age were included after detailed history, clinical examination and informed consent. Females with known medical and surgical contraindications to Hysteroscopy, suspected endocrinological abnormality, genital Infections and TB, any evidence of genital malignancy and unhealthy cervix on PS were excluded from the study.SIS was done in 124 females using Crooke's cannula. The use of Crooke's cannula enabled us to visualise the cervix and its pathologies as well, because it lacks the conventional bulb which needs to be inflated, as in case of a foley's catheter, which obliterates the cervical canal. In 2 cases, SIS could not be performed due to cervical stenosis and 3 others didn't turn up for hysteroscopy. Thus, 121 cases underwent DH under GA the next day, using a $7 \mathrm{~mm} 30^{\circ}$ telescope and normal saline as distension medium. Appropriate information was obtained and diagnosis was made on the basis of the 2 procedures. The results were compared and analysed.

\section{Criteria for Diagnosis in Saline Infusion Sonography}

1. Normal Endometrium: Normal looking endometrium with uniform thickness all around within the normal range.

2. Endometrial hyperplasia: Diffuse and irregularly thickened endometrium, without any breech in surface, with intact endo-myometrial interface; Thickness $>10 \mathrm{~mm}$.
3. Endometrial Polyps: Not as round and regular in outline as fibroid polyp; Smooth margined echogenic mass with homogenous echotexture; Sway with the movement of the fluid in cavity.

4. Endometritis: Bright echogenic foci with irregular surface.

5. Submucous fibroids: Solid round structures of mixed echogenicity that cause bulge or protrusion of the endometrium; Do not move with medium.

6. Intrauterine adhesions: Threadlike immobile strands.

7. Subseptate uterus/ bicornuate uterus.

\section{RESULTS}

In our study, we found that the maximum no. of patients were in the age group of 38yrs and above (45\%). Most of the patients $(86 \%)$ included in the study were Hindus while the rest were Muslims. But no statistical difference was found between the complains and the diagnosis on the basis of religion. More than half of the patients (53\%) were either illiterate or were just literate enough to write their own names. While only about $16 \%$ of the study population was graduate.

Irregular acyclic bleeding (Metrorrhagia) and Polymenorrhoea were the most common complain of the patients $(69 \%)$ followed by excessive bleeding and passage of clots (54\%) and prolonged periods $(30 \%)$ together may be grouped as menorrhagia. Post coital bleeding was quite uncommon and was complained of by only 5 patients (in absence of any local vaginal or cervical pathology on PS).

Almost one fifth of the patients attending the OPD with complain of AUB, had been suffering from the same for more than a year. And almost $12 \%$ had been bearing the brunt of the condition for almost 2 years and more, reflecting the state of negligence for the health and well being of a female in our society. About $53 \%$ of the patients were vigilant enough to seek advice within a few months of beginning of the problem. This mostly corresponded to the educated class of the society.

Total 121 cases underwent both SIS as well as hysteroscopy. There were 62 cases in which SIS could identify pathology while 74 such cases were identified by hysteroscopy. Out of the 29 cases of Endometrial polyps (EP) diagnosed by hysteroscopy, SIS identified 24 cases. Similarly, SIS diagnosed 12 cases of Submucous fibroids (SMF) and 12 cases of Endometrial Hyperplasia (EH) against 16 cases each diagnosed by hysteroscopy. On the other hand, SIS could not identify even a single case of endometrial carcinoma (EC) and uterine adhesions $(\mathrm{ADH})$.

\section{DISCUSSION}

The most common age group suffering from abnormal uterine bleeding is the Perimenopausal age (38-48yrs) 
which constituted about $45 \%$ of the study subjects. There was no correlation between the woman's religion and education status with the cause and pathology of AUB, although it was found that the more educated women tend to be more vigilant and cautious towards their menstrual patterns, thus seeking medical advice earlier in case of disturbed menstrual molimina. 65 women seeked medical advice within 3-7 months of AUB while 25 had been bearing the brunt of the condition for almost 2 years and more, reflecting the state of negligence for the health and well being of a female in our society.

Irregular bleeding and polymenorrhoea (69\%) was the most common form of AUB encountered in the outdoor clinics followed by menorrhagia (54\%). Post coital bleeding as a form of AUB, was the least common, in absence of any gross cervical pathology.

Patients with previous history of hormonal intake in any form (OCPs, Progesterone etc) tend to have a propensity to AUB but the relation was statistically insignificant. It was more attributed to erratic use and irregular intake of hormones that cause changes in menstrual pattern.

Endometrial polyps and submucous myomas are the 2 pathologies found in women of almost all ages while endometrial hyperplasia and endometrial carcinoma predominantly affect women of higher ages. Both these pathologies are the major causes of polymenorrhoea and menorrhagia in females of reproductive age.

The degree of anemia correlated well with the amount of bleeding and the chronicity of the problem. 58 women had $\mathrm{Hb}$ level between $7-9 \mathrm{gm} \%$, while 13 women had $<7 \mathrm{gm} \% \mathrm{Hb}$.

Total 121 cases underwent both SIS as well as hysteroscopy. There were 62 cases in which SIS could identify a pathology while 74 such cases were identified by hysteroscopy. Out of the 29 cases of Endometrial polyps (EP) diagnosed by hysteroscopy, SIS identified 24 cases. Similarly, SIS diagnosed 12 cases of Submucous fibroids (SMF) and 12 cases of endometrial hyperplasia (EH) against 16 cases each diagnosed by hysteroscopy, thereby missing a few cases. Thus there was $83 \%$ agreement between SIS and hysteroscopy in the diagnosis of EP, $75 \%$ for SMF and $75 \%$ for EH. This was however, much less as compared to $91 \%$ agreement demonstrated by Aslam et $\mathrm{al}^{2}$ in their study where they compared transvaginal sonography and saline contrast sonohysterography in women with abnormal uterine bleeding with hysteroscopy and histopathology (Table 1,2).

Table 1: Distribution of various pathologies diagnosed by SIS and hysteroscopy.

\begin{tabular}{|c|c|c|c|c|c|c|c|c|c|}
\hline & \multicolumn{9}{|c|}{ Hysteroscopy } \\
\hline SIS & ADH & EC & EH & ENDO & EP & MA & NAD & SMF & Grand Total \\
\hline $\mathrm{EH}$ & & & 12 & & & & & & 12 \\
\hline ENDO & & & & 2 & & & 2 & & 4 \\
\hline $\mathrm{EP}$ & & 3 & 1 & & 20 & & & & 24 \\
\hline MA & & & & & 5 & 5 & & & 10 \\
\hline NAD & 2 & & 3 & 1 & 4 & & 44 & 5 & 59 \\
\hline SMF & & & & & & & 1 & 11 & 12 \\
\hline Grand Total & 2 & 3 & 16 & 3 & 29 & 5 & 47 & 16 & 121 \\
\hline
\end{tabular}

Abbreviations: ADH - Adhesions, EC - Endometrial carcinoma, EP - Endometrial Polyp, EH - Endometrial Hyperplasia, MA Mullerian Anomaly, SMF - Submucous Fibroid, ENDO - Endometritis, NAD - No abnormality detected

On the other hand, SIS could not identify even a single case of endometrial carcinoma (EC) and uterine adhesions $(\mathrm{ADH})$. This was in agreement with the study conducted by Epstein, Ramirez et $\mathrm{al}^{3}{ }^{3} 2001$ that concluded that Hysteroscopy was superior to both SIS and conventional ultrasound in discriminating between benign and malignant lesions (Table 1,2).

Mullerian anomalies (MA) like bicornuate uterus, septate uterus etc was over diagnosed by SIS as even a fundal polyp or fundal fibroid was interpreted on SIS as septa. 59 cases $(49 \%)$ were found to have no abnormality on SIS while on hysteroscopy, the no. of cases was $47(39 \%)$.

According to our study, SIS was found to be most sensitive $(100 \%)$ for detection of Mullerian abnormalities (MA) with a specificity of $95.7 \%$, a negative predictive value of $100 \%$ but a PPV of only $50 \%$. It is found 
to be least sensitive (67\%) for endometritis (ENDO) (Table 3).

Table 2: Pathologies detected by SIS and hysteroscopy.

\begin{tabular}{|lllll|}
\hline Diagnosis & \multicolumn{2}{c|}{ Diagnosis of SIS } & \multicolumn{2}{c|}{$\begin{array}{l}\text { Diagnosis of } \\
\text { Hysteroscopy }\end{array}$} \\
\cline { 2 - 5 } & No. & $\%$ & No. & $\%$ \\
\hline EP & 24 & 19.84 & 29 & 23.97 \\
\hline SMF & 12 & 9.92 & 16 & 13.22 \\
\hline EH & 12 & 9.92 & 16 & 13.22 \\
\hline MA & 10 & 8.26 & 5 & 4.13 \\
\hline ENDO & 7 & 5.78 & 3 & 2.48 \\
\hline EC & 0 & 0.00 & 3 & 2.48 \\
\hline ADH & 0 & 0.00 & 2 & 1.65 \\
\hline NAD & 59 & 48.76 & 47 & 38.84 \\
\hline Total & 121 & 100.00 & 121 & 100.00 \\
\hline
\end{tabular}

SIS had a sensitivity of $75 \%$ for diagnosing endometrial hyperplasia (EH) with specificity, NPV and a PPV of $100 \%$, $100 \%$ and $96.3 \%$ respectively. This was much less as compared to the results obtained by Valenzano et $\mathrm{al}^{4}$, SIS had a $100 \%$ sensitivity and $100 \%$ specificity in diagnosis of EH. But was better as compared to results obtained by Grigoris F Grimbizis et $\mathrm{al}^{5}$ in Greece. He found a sensitivity of $29 \%$ and a specificity of $99 \%$ for endometrial hyperplasia. Similarly our results also matched to those obtained by Krampl et al $^{6}$ who showed SIS had sensitivity of $33 \%$ and specificity of $92 \%$ in diagnosis of endometrial hyperplasia and carcinoma (Table 3 ).

The sensitivity and specificity for endometrial polyps (EP) in our study is $69 \%$ and $95.7 \%$, and that for sub mucus fibroids is $68.75 \%$ and $99 \%$ respectively. This was in contrast to the findings suggested by Bernard et $\mathrm{al}^{7}$ who studied 109 premenopausal and 53 postmenopausal women and determined the sensitivity of $87.8 \%$ and $89.6 \%$ and specificity of $90.7 \%$ and $95 \%$ for diagnosis of Polyps and Submucous Myomas, respectively. Ramirez et $\mathrm{al}^{8}$ obtained a sensitivity of $79.4 \%$ for detection of EP and SMF (Table 3).

Table 3: Efficiency of saline infusion sonography for detection of causal pathology of abnormal uterine bleeding.

\begin{tabular}{|lllllllll|}
\hline & Sensitivity & Specificity & \multicolumn{2}{c}{ PPV } & \multicolumn{2}{c|}{ NPV } \\
\hline & & $\%$ & & $\%$ & & $\%$ & & $\%$ \\
\hline EH & $12 / 16$ & 75 & $105 / 105$ & 100 & $12 / 12$ & 100 & $105 / 109$ & 96.3 \\
\hline ENDO & $2 / 3$ & 66.67 & $116 / 118$ & 98.3 & $2 / 4$ & 50 & $116 / 117$ & 99 \\
\hline EP & $20 / 29$ & 69 & $88 / 92$ & 95.7 & $20 / 24$ & 83 & $88 / 97$ & 90 \\
\hline MA & $5 / 5$ & 100 & $111 / 116$ & 95.7 & $5 / 10$ & 50 & $111 / 111$ & 100 \\
\hline NAD & $44 / 47$ & 93.2 & $63 / 78$ & 80.8 & $44 / 59$ & 75 & $59 / 62$ & 95 \\
\hline SMF & $11 / 16$ & 68.75 & $104 / 105$ & 99 & $11 / 12$ & 92 & $104 / 109$ & 95.5 \\
\hline ADH & $0 / 2$ & 0 & $119 / 119$ & 100 & - & - & $119 / 121$ & 98.3 \\
\hline
\end{tabular}

Table 4: Diagnostic efficacy of SIS.

\begin{tabular}{|l|c|}
\hline & $\begin{array}{c}\text { Diagnostic Efficacy (true positives }+ \\
\text { true negatives)/ total study subjects }\end{array}$ \\
\hline EH & $96.69 \%$ \\
\hline ENDO & $97.52 \%$ \\
\hline EP & $89.25 \%$ \\
\hline MA & $95.86 \%$ \\
\hline SMF & $95.04 \%$ \\
\hline ADH & $98.34 \%$ \\
\hline
\end{tabular}

Table 5: Positive diagnosis: Hysteroscopy v/s SIS.

\begin{tabular}{|lll|}
\hline Test & No & $\%$ \\
\hline Hysteroscopy & 74 & 61.16 \\
\hline SIS & 62 & 51.24 \\
\hline
\end{tabular}

Overall, about $61 \%$ of the cases of AUB were diagnosed to have some abnormality by hysteroscopy as compared to $51 \%$ by SIS. 
Table 6: Overall efficacy of saline infusion sonography.

\begin{tabular}{|lllll|}
\hline & Sensitivity & Specificity & PPV & NPV \\
\hline SIS & $67.56 \%$ & $93.6 \%$ & $80.6 \%$ & $74.57 \%$ \\
\hline
\end{tabular}

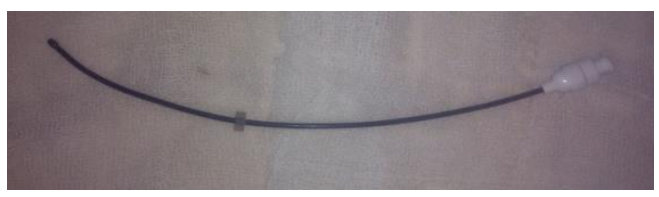

Figure 1: Crooke's cannula.

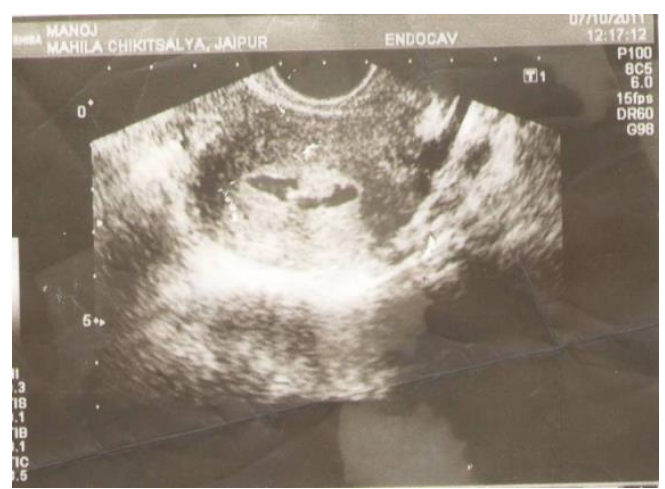

Figure 2: Endometrial polyp in saline infusion sonography.

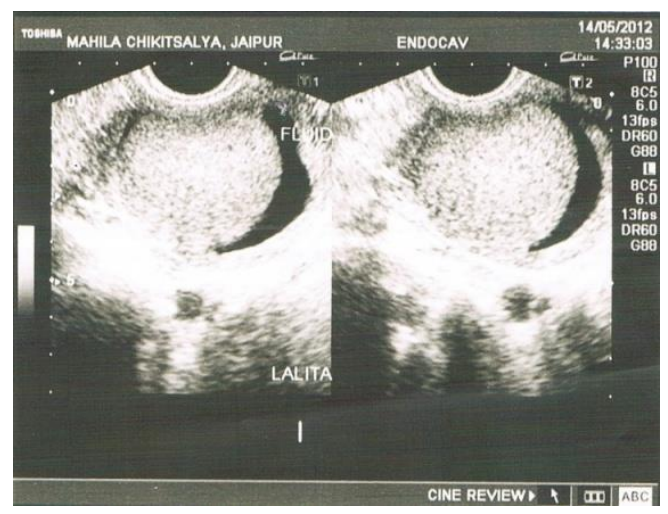

Figure 3: Submucous fibroid in saline infusion sonography.

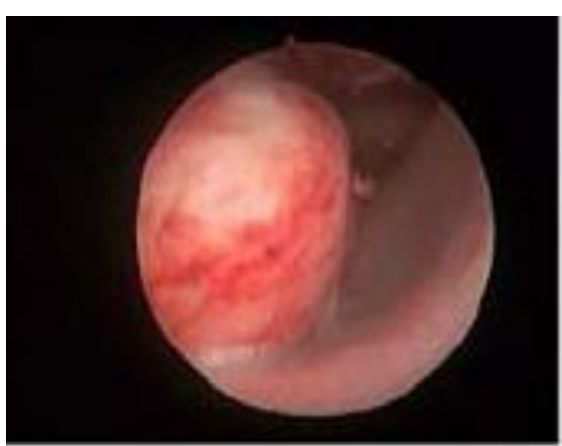

Figure 4: Hysteroscopic view of submucous fibroid.

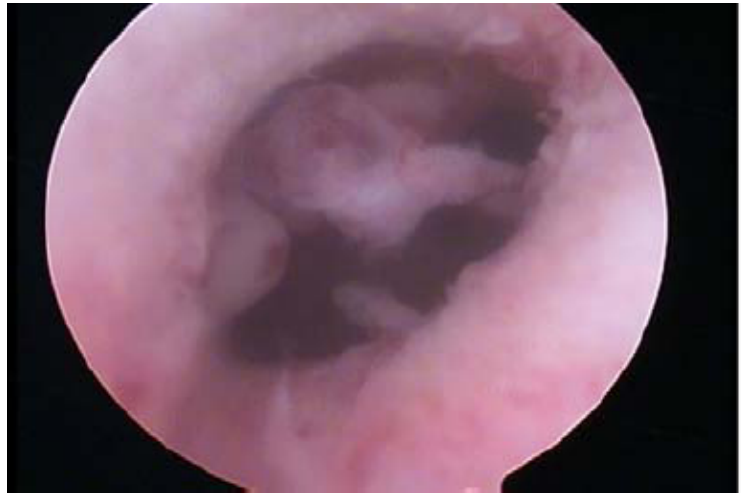

Figure 5: Hysteroscopic view of endometrial polyps.

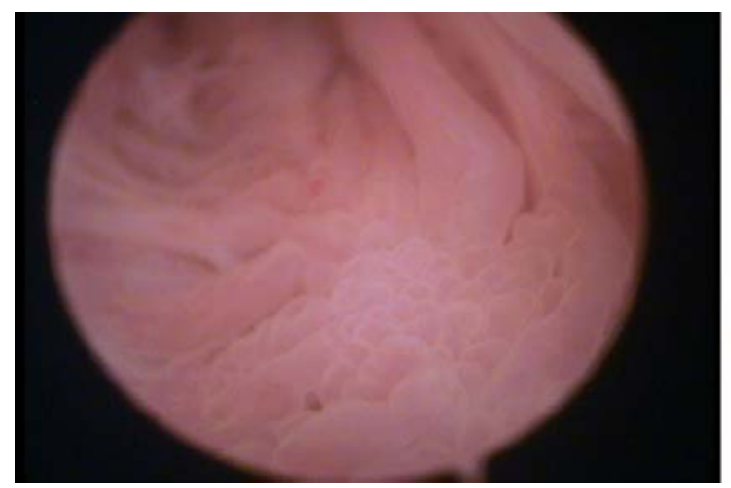

Figure 6: Hysteroscopic view of secretory endometrium.

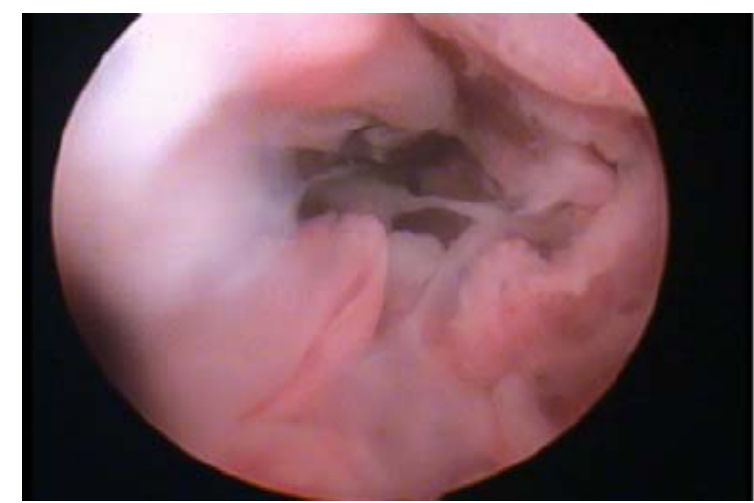

Figure 7: Hysteroscopic view of endometrial hyperplasia.

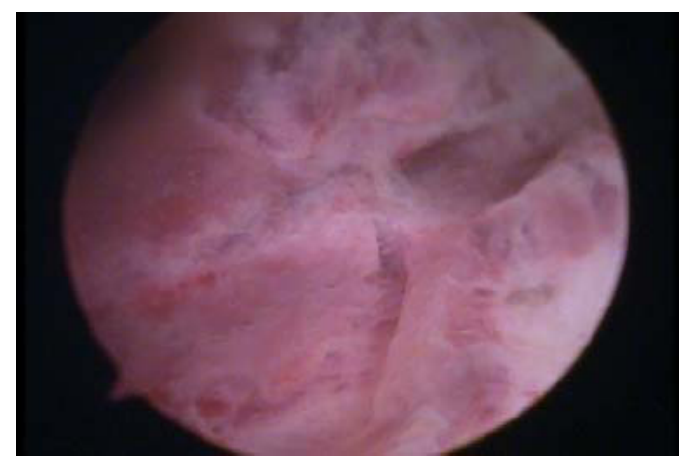

Figure 8: Hysteroscopic view of endometrial cancer. 
This study showed that the sensitivity of SIS is greatest (100\%) for Mullerian anomalies, $75 \%$ for endometrial hyperplasia, $69 \%$ for endometrial polyps, $68.75 \%$ for submucous myomas and $66.67 \%$ for endometritis. The specificity is greatest for endometrial hyperplasia and adhesions (100\%), 99\% for submucous myomas, $98.3 \%$ for endometritis, $95.7 \%$ for endometrial polyps and mullerian anomalies. It has a $100 \%$ positive predictive value for endometrial hyperplasia, $92 \%$ and $83 \%$ for submucous myomas and endometrial polyps respectively. Thus the final diagnostic efficacy of SIS for endometrial hyperplasia, endometritis, endometrial polyps, mullerian abnormalities, submucous fibroid and adhesions was $96.69 \%, 97.53 \%, 89.25 \%, 95.86 \%, 95.04 \%$ and $98.34 \%$ respectively.

The overall sensitivity, specificity, positive predictive value (PPV) and negative predictive value (NPV) of SIS in our setting is $67.56 \%, 93.6 \%, 80.6 \%$ and $74.5 \%$ respectively (Table 6). There have been conflicting results in for the efficiency of SIS. Wildrich et $\mathrm{al}^{9}$ in 1996 studied evaluated 130 patients with abnormal bleeding by SIS as well as hysteroscopy and determined that for all findings combined, sensitivity was $96 \%$ and specificity was $88 \%$. Lynne Rogerson et al $^{10}$ studied 117 women of AUB an reported a sensitivity of $85.2 \%$, specificity of $87.3 \%$, PPV and NPV of $74.2 \%$ and $93.2 \%$ respectively. In India, Pasrija, Trivedi, et al $^{11}$ found the sensitivity, specificity, positive predictive value and negative predictive value of SIS to be $94.4 \%, 88.5 \%, 91.4 \%$ and $92 \%$ respectively.

\section{CONCLUSION}

On concluding the present study, there was a variation in sensitivity and specificity of the procedure in relation to our co workers, though SIS turned out to be an easy procedure. We can affirm that, in a Government setup in India like ours, where the number of patients is large and the resources are limited, SIS is an easier procedure. Though is not very sensitive, but it has a good positive and negative predictive value, and can certainly reduce and avoid the unnecessary hysteroscopies performed and the morbidity related to it. If there is any ambiguity of diagnosis, hysteroscopy may be performed. It cannot replace diagnostic hysteroscopy on the whole.

Funding: No funding sources

Conflict of interest: None declared

Ethical approval: The study was approved by the Regional Committee for Medical Ethics and Research

\section{REFERENCES}

1. Richman et al., Fallopian tube patency as assessed by ultrasonogram following fluid injection. Rad. 1984;152:507-510.

2. Aslam M, Ijaz L, Tariq S, Shafqat K, Meher-UnNisa, Ashraf R, Kazmi T. Comparison of transvaginal sonography and saline contrast sonohysterography in women with abnormal uterine bleeding: correlation with hysteroscopy and histopathology. Int J Health Sci (Qassim). 2007 Jan;1(1):17-24.

3. Epstein E, Ramirez A, et al. Transvaginal sonography, saline contrast sonohysterography and hysteroscopy for the investigation of women with abnormal bleeding and endometrium $>5 \mathrm{~mm}$. Ultrasound Obstet Gynecol 2001 Aug;18(2):157-62.

4. Valenzano MM, Lijoi D, Mistrangelo E, Fortunato $\mathrm{T}$, Costantini S, Ragni $\mathrm{N}$. The value of sonohysterography in detecting intracavitary benign abnormalities. Arch Gynecol Obstet. 2005 Oct;272(4):265-8.

5. Grigoris F. Grimbizis, Eftychia Anagnostou, Efstratios Asimakopoulos, Panagiotis Stamatopoulos, Basil C. Tarlatzis Papageorgiou General Hospital, Thessaloniki, Greece "A prospective comparison of transvaginal ultrasound, saline infusion sonohysterography, and diagnostic hysteroscopy in the evaluation of endometrial pathology" Fertility and Sterility 2010.

6. Krampl E; Bourne T; hurlen-Solbakken H; Istre O. Transvaginal Ultrasonography, sonohysterography and operative hysteroscopy for the evaluation of abnormal uterine bleeding. Acta Obstet Gynecol Scandinavia $2001 \mathrm{Jul} ; 80(7): 616-22$.

7. Bernard JP, et al. Saline contrast sonohysterography as first-line investigation for women with uterine bleeding. Ultrasound in Obstetrics and Gynecology, 1997;19121-5.

8. Epstein E; Ramirez A, et al. Transvaginal sonography, saline contrast sonohysterography and hysteroscopy for the investigation of women with abnormal bleeding and endometrium > $5 \mathrm{~mm}$. Ultrasound Obstet Gynecol 2001 Aug;18(2):157-62.

9. Wildrich T, Bradley LD, Mitchinson AR Collins RL. Comparison of saline infusion sonography with office hysteroscopy for the evaluation of the endometrium. Am J Obstet Gynecol. 1996 Apr; 174(4):1327-34.

10. Lynne Rogerson, Jane Bates, Mike Weston, Sean Duffy. A comparision of outpatient hysteroscopy with saline infusion hysterosonography. BJOG, July 2002.

11. Shikha Pasrija, Shubha Sagar Trivedi, Mahinder Kaur Narula. Prospective study of saline infusion sonohysterography in evaluation of perimenopausal and postmenopausal women with abnormal uterine bleeding. Journal of Obstetrics and Gynaecology Research 2004;30(1):27-33.

DOI: $10.5455 / 2320-1770$. ijrcog20131209

Cite this article as: Sharma M, Joshi S, Sharma A, Nagar O, Khuteta R, Bagarahatta M. Role of saline infusion sonography in abnormal uterine bleeding. Int J Reprod Contracept Obstet Gynecol 2013;2:533-8. 